\title{
MORTALIDADE MATERNA NO MUNICÍPIO DO RIO DE J ANEIRO: MAGNITUDE E DISTRIBUIÇÃO
}

\author{
Maternal morbidity in the district of Rio de Janeiro: magnitude and \\ distribution \\ La mortalidad materna en el municipio de Rio de J aneiro: magnitud y \\ distribuición
}

Enirtes Caetano Prates Melo ${ }^{1}$

Virginia Maria de Azevedo Oliveira Knupp ${ }^{2}$

\section{RESUMO}

A mortalidade materna é um evento traçador da assistência por ser evitável em 92\% dos casos. Trata-se de um estudo descritivo de base populacional que analisou as declarações de óbito das mulheres de 10 a 49 anos no Município do Rio de Janeiro, 19962004. Foram utilizados os dados do Sistema de Informações sobre Mortalidade, processados e mapeados através do TabWin. Verificou-se o predomínio do óbito materno entre mulheres solteiras e mulheres com 4 a 7 anos de estudo. A Razão de Mortalidade Materna permanece alta no município. Dois aglomerados chamam atenção na distribuição espacial dos óbitos maternos. 0 primeiro abrange a Zona Oeste e apresenta uma Razão de Mortalidade Materna muito alta. 0 segundo situa-se ao longo do subúrbio da Leopoldina e concentra uma mortalidade alta.

Palavras-chave: Mortalidade Materna. Saúde da Mulher. Sistemas de Informação. Qualidade da Assistência à Saúde.

\begin{abstract}
Maternal mortality is an event that reflects the assistance provided because it deals with unavoidable deaths in $92 \%$ of the cases.

This is a population-based study that analyzed the deaths certificates of women between 10 to 49 years in a Rio de Janeiro district, from 1996 to 2004. The data used was obtained from the Mortality Information System, processed and mapped through TabWin. The predominance of the deaths verified was among the group of single women and women with 4 to 7 years of schooling. . The maternal mortality rate is still high in the city. On the spatial distribution two groups call attention. The first one includes the West Zone, showing a very high Maternal Mortality Rate. The second one is located along the the Leopoldina suburb and concentrates a high mortality rate.
\end{abstract}

Keywords: Maternal mortality, Women's Health, Information Systems, Quality of Health Care

\section{Resumen}

La mortalidad materna es un acontecimiento que refleja de la asistencia prestada por expresar una muerte evitable en el $92 \%$ de los casos. Se trata de un estudio descriptivo de base poblacional que analizó los certificados de defunción de mujeres de 10 a 49 años, en la ciudad del Río de Janeiro, de 1996 a 2004. Fueron utilizados los datos del Sistema de Informaciones sobre Mortalidad, que fueran procesados y mapeados a través del TabWin. Se contató el predominio de la muerte materna en el grupo de mujeres solteras, con entre 4 a 7 años de formación. El indice de Mortalidad Materna sigue siendo alta en la ciudad. Dos aglomerados llaman la atención en la distribución espacial de las muertes maternas. El primero en la Zona Oeste que presenta indice de mortalidad materna muy alto. El segundo se situa a lo largo de la region de la Leopoldina donde se concentra un indice de mortalidad materna alto.
Palabras-claves: : Mortalidad Materna, Salud de la mujer, Sistemas de información, Calidad de la Asistencia a laSalud.

${ }^{1}$ Enfermeira, Doutora em Ciências pela Escola Nacional de Saúde Pública - FIOCRUZ. Professora Adjunta do Departamento de Enfermagem em Saúde Pública da Escola de Enfermagem Alfredo Pinto da Universidade Federal do Estado do Rio de Janeiro e-mail: enirtes@globo.com² Enfermeira, Mestranda do Programa de Pós-Graduação - Mestrado em Enfermagem da Escola de Enfermagem Alfredo Pinto - UNIRIO - Rio de Janeiro - RJ. 


\section{INTRODUÇ̃̃O}

Observa-se nas últimas décadas um declínio acentuado da mortalidade materna e infantil nos países desenvolvidos ${ }^{1,2}$. A Razão de Mortalidade Materna (RMM) e o coeficiente de mortalidade infantil são reconhecidos não só como indicadores das condições de saúde da população, como também eventos que refletem as condições gerais de vida, sendo considerados sínteses da qualidade de vida e do nível de desenvolvimento de uma população. A RMM mede o risco de uma mulher morrer por uma causa relacionada ao ciclo gravídico-puerperal.

A Organização Mundial de Saúde define a morte materna como aquela que ocorre durante a gestação ou no período de 42 dias após o término da gestação, independente da duração ou da localização da gravidez, por qualquer causa relacionada com ou agravada pela gravidez, ou por medidas voltadas a ela, porém não por causas acidentais ou incidentais. A morte materna é um evento traçador da assistência. Representa um grande problema de saúde pública por se tratar de uma morte evitável em $92 \%$ dos casos ${ }^{3,4}$.

Estima-se que 529 mil mulheres morram anualmente em decorrência de complicações relacionadas ao ciclo gravídicopuerperal. No Brasil, a RMM em 1997 foi de 72 óbitos por 100.000 nascidos vivos. Ao longo dos anos as políticas públicas voltadas para esse grupo específico não têm sido suficientes para reduzir a morte materna, que permanece elevada. Em 2004, a RMM foi de 54,2 óbitos por 100.000 nascidos vivos, razão ainda considerada alta de acordo com parâmetros estabelecidos pela Organização Pan-Americana de Saúde.

Fatores sócio-demográficos culturais maternos (idade, escolaridade, renda, estado civil) têm marcado influência no acesso aos serviços de atendimento à gravidez e ao nascimento, mesmo nos países desenvolvidos que apresentam melhores indicadores globais de saúde materno-infantil, mostrando que as possibilidades de utilização dos recursos disponíveis são determinadas pela inserção social ${ }^{5,6}$.

Considerando que a possibilidade de intervenção no perfil da mortalidade materna e infantil parece deslocar-se cada vez mais para a esfera dos serviços de saúde, especialmente os médico-assistenciais, e que o acesso à assistência médica de qualidade tem papel fundamental na determinação da mortalidade, deve-se investigar a existência de desigualdades no acesso a tais serviços. 0 padrão de localização dos serviços e da distribuição da população por eles atendida permite identificar as redes que os conectam, bem como lacunas na distribuição dos serviços, e variações nas distâncias que os indivíduos devem percorrer para obter atendimento.

No Rio de Janeiro, vários estudos indicam que populações socialmente desfavorecidas apresentam índices de mortalidade mais altos para diversas causas de óbito. A deterioração das condições de saúde nas áreas que agregam alta proporção de residentes em favela sugere uma pior condição de saúde e um risco aumentado para o óbito $7,8,9,10$
Este estudo tem como objetivo analisar a evolução da Razão de Mortalidade Materna e sua distribuição espacial no Município do Rio de Janeiro.

\section{METODOLOGIA}

Trata-se de um estudo descritivo de base populacional que analisou as Declarações de Nascidos Vivos (DNV) e as Declarações de Óbito (DO) das mulheres na faixa etária de 10 a 49 anos, residentes no Município do Rio de Janeiro, no período de 1996 a 2004.

Foram utilizadas as bases de dados referentes aos Sistemas de Informações sobre Nascidos Vivos (SINASC) e sobre Mortalidade (SIM), disponibilizadas pela Secretaria Municipal de Saúde do Rio de Janeiro após aprovação do estudo pelo Comitê de Ética em Pesquisa do Hospital Universitário Gaffrée e Guinle. 0 georreferenciamento dos nascimentos e dos óbitos maternos foi efetuado com base na Região Administrativa (RA) de residência da mãe e no endereço do estabelecimento de saúde onde ocorreram os eventos, que consta na DNV e na DO.

No SIM foram selecionados os casos de óbito em mulheres residentes no município, na faixa etária de 10 a 49 anos, no período de 1996 a 2004, e analisados aqueles cuja causa básica foi morte materna (Código 088-091 da Classificação Estatística Internacional de Doenças e Problemas Relacionados à Saúde - CID-10). Foram analisadas na D0 as seguintes variáveis: causa do óbito materno, estado civil, faixa etária, escolaridade de estudo e raça/etnia.

o Município do Rio de Janeiro está dividido administrativamente em 153 bairros, agregados em 30 Regiões Administrativas (RA). As RAs compõem cinco Áreas de Planejamento (AP). A AP 1 configura a zona mais antiga e central da cidade. As APs 2.1 e 2.2 correspondem respectivamente à Zona Sul e à Zona Norte. As 3.1, 3.2 e 3.3 correspondem ao subúrbio da Central e Leopoldina, além da Ilha do Governador. As APs 4, 5.1, 5.2 e 5.3 correspondem à Zona Oeste.

As APs 1, 2, e 3 apresentam alta densidade populacional e são dotadas de infra-estrutura urbana básica. AAP 3 responde por cerca da metade dos habitantes de favelas. As APs 4 e 5 apresentam baixa densidade e carência de infra-estrutura básica. A AP 2.1 apresenta o nível de renda mais elevado, e a Zona Oeste, o mais baixo.

A RMM foi calculada para o período de 1996 a 2004. No caso especificamente da dinâmica espacial da RMM, optou-se por concentrar a análise do padrão espacial e a análise dos fluxos no período de 2002 a 2004. Essa decisão se deu em função da qualidade do preenchimento do campo relativo ao endereço de residência e ocorrência do evento na DO.

A Morte Materna Obstétrica é classificada em direta e indireta. A Direta é aquela que decorre de complicações obstétricas durante a gravidez, o parto ou o puerpério devido 
às intervenções, omissões, tratamento incorreto ou a uma série de eventos resultantes de qualquer uma dessas causas. $A$ Indireta é aquela resultante de doenças que existiam antes da gestação ou que se desenvolvem durante esse período, não provocadas pelas causas obstétricas diretas, mas agravadas pelos efeitos fisiológicos da gravidez ${ }^{1}$.

Os mapas de fluxos com flechas permitem identificar 0 volume de "tráfego" entre local de residência (RA de residência) e o local de assistência (RA de ocorrência), desde que identificados nos Sistemas de Informação em Saúde (SIS). A largura de cada flecha é proporcional ao fluxo. 0 padrão das setas permite verificar as distâncias percorridas em linha reta pela população na busca pela assistência, bem como identificar anomalias e particularidades neste fluxo, áreas que concentram serviços de saúde e áreas com vazios sanitários. Nesse estudo trabalhou-se somente com a origem e os destinos finais, uma vez que o percurso não está disponível nos SIS. É importante observar que este tipo de mapa permite identificar o volume de deslocamentos, questão importante para a compreensão de padrões de risco para a morte materna.

0 processamento e mapeamento dos dados foram realizados através sistema TAB para Windows - TabWin, programa de domínio público, desenvolvido pelo DATASUS.

\section{RESULTADOS}

OSIM registrou 511 óbitos maternos, no período de 1996 a 2004, no Município do Rio de Janeiro. A RMM apresentou uma queda em 1997 (49,7 óbitos por 100.000 nascidos vivos) e um incremento em 2001 (67,8 óbitos por 100.000 nascidos vivos) (Figura 1). A RMM permaneceu elevada, variando de média, em 1997, a alta, no período entre 1998 e 2004, segundo a classificação proposta pela Organização PanAmericana de Saúde.

\section{Figura 1- Distribuição da Razão de Mortalidade Materna no Município do Rio de Janeiro, 1996 a 2004.}

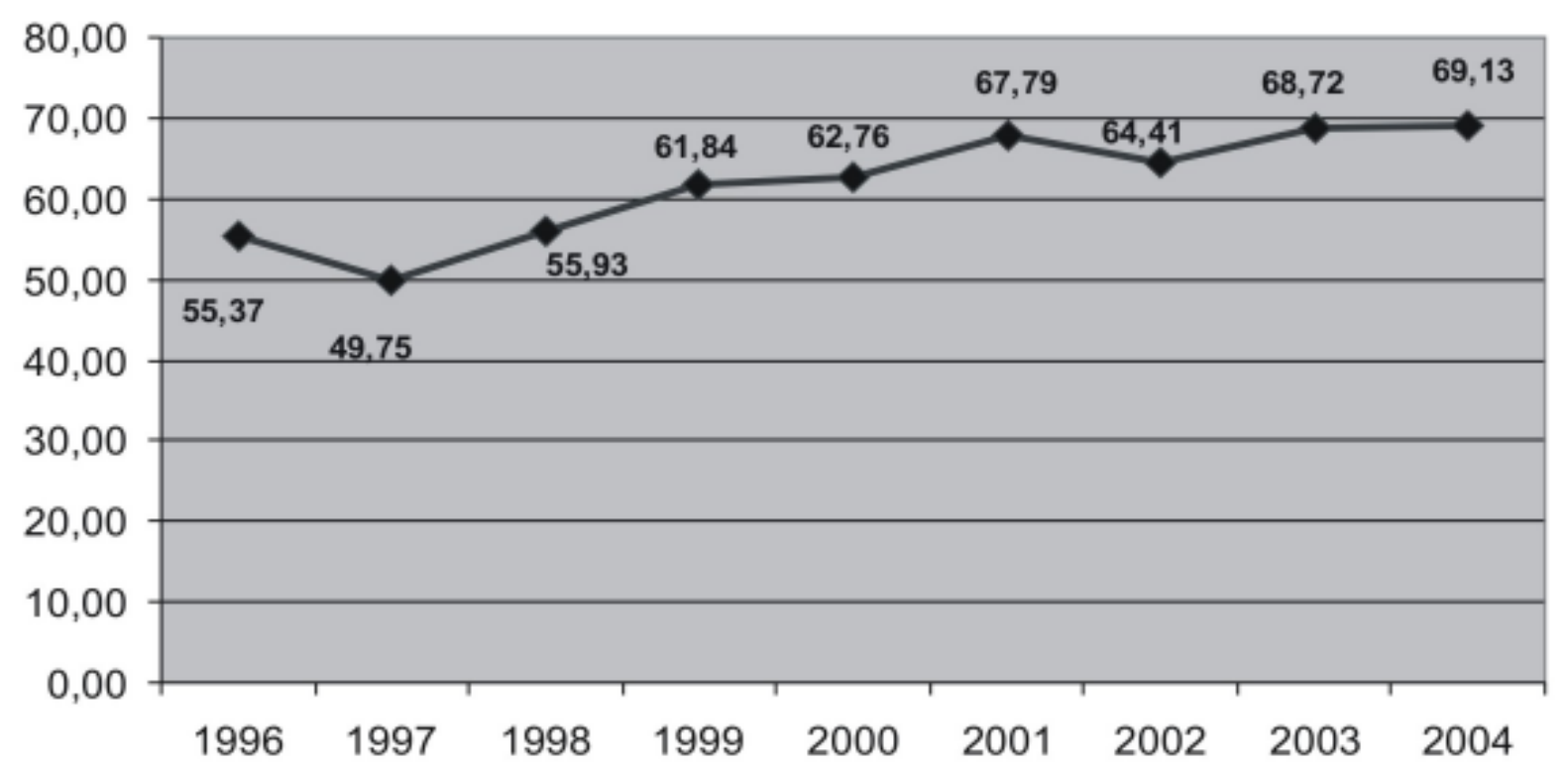

Observou-se um predomínio de óbitos maternos, em 2004, entre mulheres solteiras (67,3\%) e na faixa etária de 30 a 39 anos (40,8\%) (Figura 2). Há uma concentração de óbitos maternos entre mulheres com 4 a 7 anos de estudo (34,9\%). Não foram observadas diferenças na distribuição dos óbitos maternos em relação à raça/etnia. 
Figura 2 - Distribuição percentual do óbito materno segundo faixa etária no Município do Rio de Janeiro, 2004.

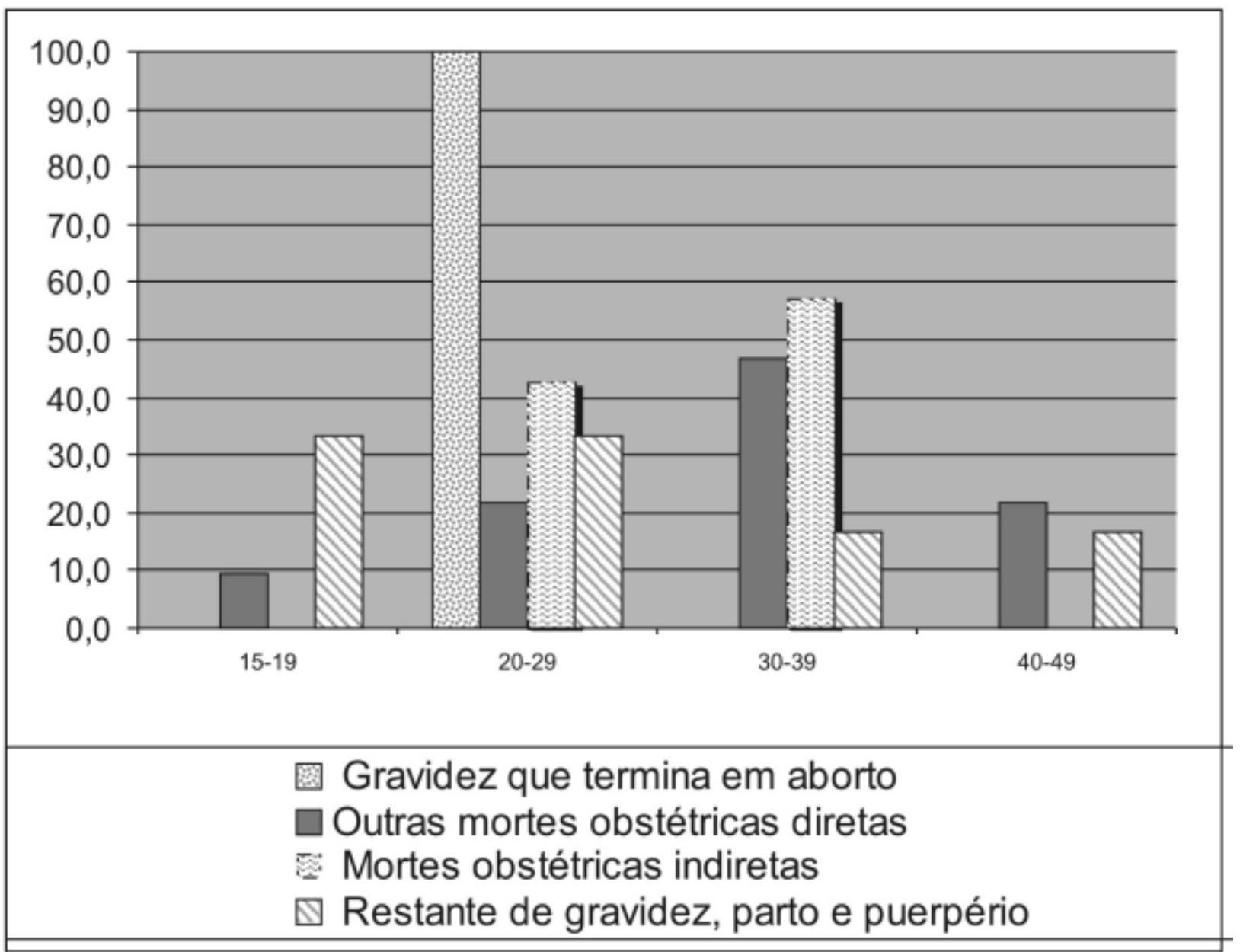

Em 2002, dois aglomerados chamam atenção na distribuição espacial dos óbitos maternos (Figura 3). 0 primeiro abrange a Zona Oeste e apresenta uma RMM considerada muito alta. 0 segundo situa-se ao longo do subúrbio da Leopoldina e concentra uma mortalidade alta.

Figura 3 - Distribuição Espacial da Razăo de Mortalidade Materna no Município do Rio de Janeiro, 2002.

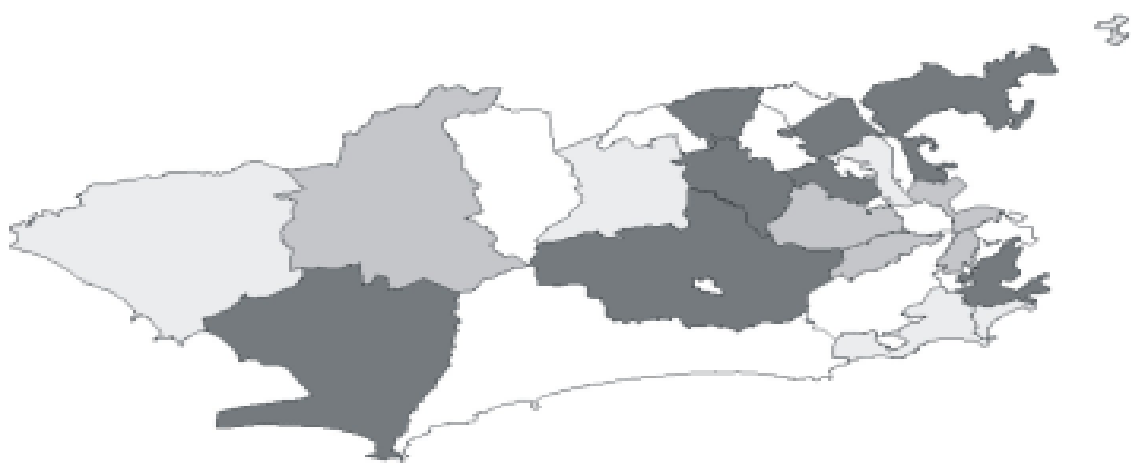


Destacam-se em 2004 dois grandes aglomerados com uma razão de óbito elevada (Figura 4). 0 primeiro merece maior destaque, abrange a Zona Oeste e apresenta uma RMM muito alta (acima de 150 mortes por 100.000 nascidos vivos). 0 segundo situa-se ao longo do subúrbio da Leopoldina e concentra uma RMM considerada alta (entre 50 e 149 mortes por 100.000 nascidos vivos).

\section{Figura 4 - Distribuição Espacial do Fluxo da Razão de Mortalidade Materna no Município do Rio de Janeiro, 2004.}

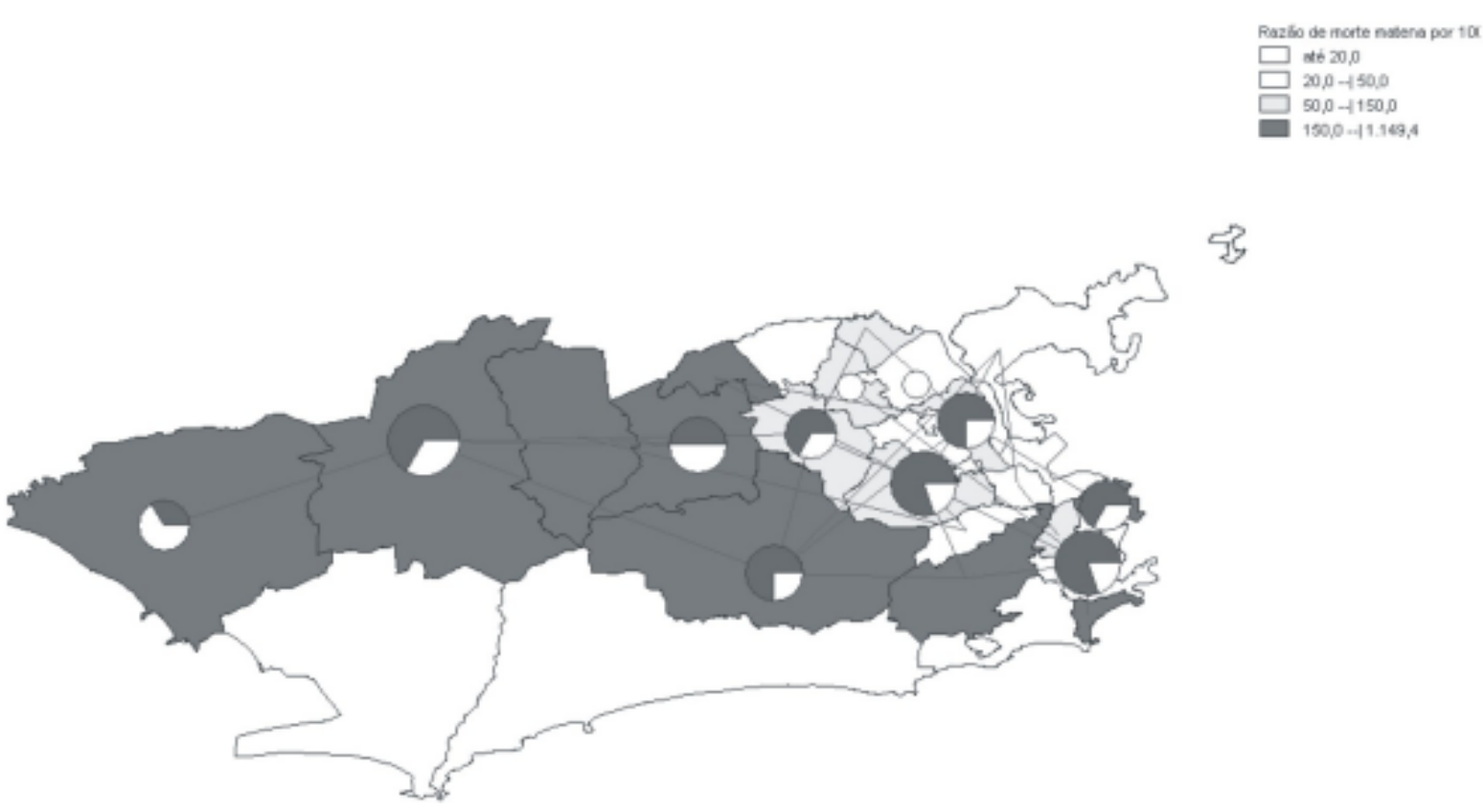

A Figura 5 permite identificar os fluxos entre a RA de residência da mulher (mãe) e a RA do hospital (maternidade) onde ocorreu o óbito. Verifica-se, no período de 1996 a 2004, um deslocamento de residentes da Zona Oeste em direção à região mais central da cidade, onde se concentram os serviços de saúde.

Figura 5 - Distribuição Espacial do Fluxo do Óbito Materno no Município do Rio de Janeiro no período de 1996 a 2004.

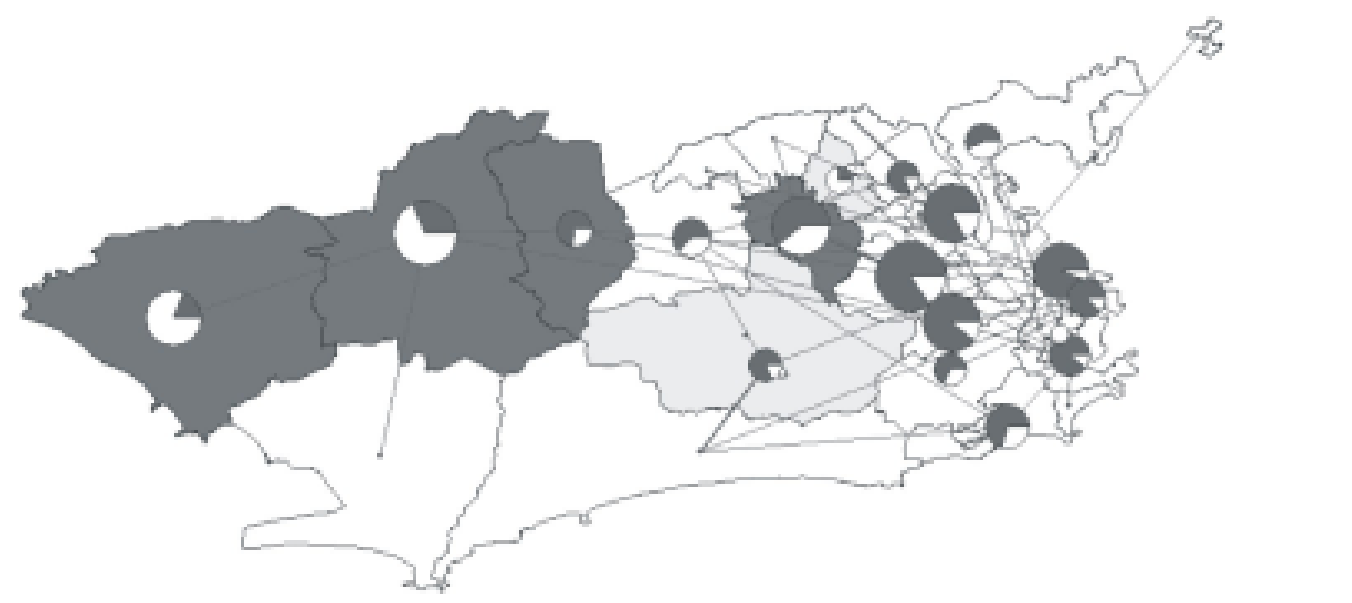




\section{DISCUSSÃO}

Os diversos estudos sobre mortalidade materna reconhecem o problema da qualidade da informação em função do sub-registro (não preenchimento da declaração de óbito) e da subinformação (preenchimento incorreto das declaraç̃̃es de óbito), mas as estatísticas de mortalidade permanecem como a única fonte de dados disponível que possibilita traçar os fatores de risco a ela associados.

A proporção elevada de óbitos maternos entre mulheres solteiras merece uma análise mais cuidadosa dos dados. Acredita-se que as variações observadas possam ser explicadas, em parte, pela flutuação aleatória deste indicador. Ainda assim, a ausência de uma rede de apoio social, da qual o companheiro participa, representa um importante fator de risco relacionado a desfechos desfavoráveis, dentre eles o adoecimento e o óbito. Nos estratos mais pobres da população, essa rede composta por familiares, amigos e parceiros pode representar a única possibilidade de suporte da mulher em condições diversas de vulnerabilidade ${ }^{11,12}$.

A concentração de óbitos maternos observada na faixa etária de 30 a 39 anos é compatível com o perfil de distribuição observado entre algumas patologias graves prevalentes durante a gestação, como a doença hipertensiva espećifica da gestação (DHEG) e o diabetes ${ }^{13}$.

A escolaridade materna deve ser vista como um proxyde classe social, uma vez que é considerada um indicador da posição social. 0 nível educacional da mãe pode ser compreendido como um fator relacionado ao perfil cultural e ao comportamento ligado aos cuidados de saúde, que têm um efeito importante na determinação da mortalidade ${ }^{14}$.

A variável raça/etnia merece uma análise mais cuidadosa dos dados, considerando que no setor saúde a formulação desse conceito é freqüentemente traduzida através de um indicador considerado precário, a cor da pele. Cabe considerar ainda que formas de identificação racial, étnica ou por ancestralidade não são suscetíveis aos critérios-padrão de cientificidade, dada a possibilidade de os indivíduos mudarem sua identidade étnico-racial ao longo do tempo e em decorrência de circunstâncias políticas e sociais ${ }^{15}$.

Uma questão que merece destaque para o planejamento do setor saúde é a distribuição, no espaço geográfico, dos serviços e de sua clientela. A falta de informação e análise sobre origem da clientela dos serviços dificulta: a investigação dos fluxos de pessoas que demandam tais serviços, conectando residência e local de atendimento; a identificação das redes estabelecidas por tais fluxos; e a delimitação de áreas de abrangência das unidades públicas, com base em sua efetiva utilização.

A RMM configura-se como um dos indicadores capazes de traduzir o estado de saúde da população feminina. 0 óbito materno persiste como relevante evento em saúde pública, estimador de iniqüidades, que demanda estudos capazes de definir sua magnitude e variações no tempo ${ }^{16,17}$. Como já era esperado, o padrão espacial de mortalidade materna apresentou uma concentração do risco de morrer nas áreas mais pobres da cidade, o que destaca a importância de investimento específico para atender às necessidades de saúde identificadas nesses locais. A delimitação de regiões com base nos fluxos que denotam a área de influência de um centro permite investigar os níveis de oferta per capita, identificando e orientando a superação de iniqüidades na distribuição dos serviços.

A RMM no Rio de Janeiro permanece elevada, variando, segundo a classificação proposta pela Organização PanAmericana de Saúde, de média a alta no período. Acredita-se que o aumento observado na RMM é explicado, em parte, em função de uma melhora na assistência, na qualidade dos dados e na intensificação das investigações realizadas através do trabalho dos Comitês de Mortalidade Materna. Diversos estudos sobre mortalidade materna reconhecem o problema da qualidade da informação em função do sub-registro e da subinformação $0^{16,17}$. No município destacam-se dois importantes aglomerados na distribuição dos óbitos maternos: um situado ao longo do subúrbio da Leopoldina e outro que abrange aZona Oeste da cidade. Alguns cuidados devem ser tomados na utilização de mapas de padrões, pois a utilização de sombreamentos e padrões de cor pode criar um "efeito visual" tendencioso. Dentre os problemas a serem considerados na construção dos mapas da distribuição dos óbitos maternos está a variabilidade aleatória da RMM, associada ao tamanho das unidades geográficas de análise. Outro problema comum é que grandes áreas de baixa densidade populacional podem predominar visualmente sobre as pequenas áreas de grande densidade populacional. A produção de mapas temáticos exige extremo cuidado, especialmente nos casos de populações com valores reduzidos nas medidas de interesse. Procedimentos de suavização são recomendados nestes casos, uma vez que reduzem a influência da flutuação aleatória das medidas, permitindo a produção de mapas mais confiáveis e a visualização de áreas que apresentam risco elevado.

0 exame dos fluxos foi afetado pelo baixo volume de óbitos maternos, o que pode determinar importantes flutuações aleatórias e interferir na detecção de padrões espaciais. Apesar desta limitação, é importante considerar a distribuição de óbitos maternos, pois os mapas parecem apontar problemas ligados à insuficiência de recursos para o atendimento dos casos de maior risco para o óbito.

Verifica-se no Rio de Janeiro um padrão de deslocamento no sentido oeste-leste, confirmando que as RAs onde há disponibilidade de leitos obstétricos funcionam como atratoras. De um modo geral, os fluxos das gestantes apontam na direção das regiões mais ricas da cidade, ligando as parturientes que residem em áreas de baixa renda a áreas onde se encontra a maior disponibilidade de serviços de saúde. Este deslocamento em direção a algumas áreas da cidade merece destaque uma vez que, em situações de urgência obstétrica, a proximidade e 
o acesso aos serviços de saúde pode determinar o desfecho materno $^{13 ; 16}$.

\section{CONCLUSÕES}

Apesar dos avanços tecnológicos e científicos e do seu caráter de evitabilidade, a mortalidade materna é, ainda hoje, um grande problema a ser enfrentado. A redução desse agravo e, indiretamente, da mortalidade infantil, requer a identificação de diferenciais de risco que permitam orientar a alocação de recursos.

Em que pese o papel do conjunto de práticas propostas para o rastreamento de condições/situações de risco durante 0 pré-natal e o parto, e com o recém-nascido no nível individual, estratégias voltadas para o contexto em que o binômio mãe-

\section{Referências}

1. World Health Organization- WHO. Maternal mortality in 2000: estimates developed by WHO, UNICEF and UNFPA. Geneva(CH); 2003.

2. Schneider MC, Castillo-Salgado C, Loyola-Elizondo E, Bacallao J, Mujica OJ, Vidaurre M, et al. Trends in infant mortality inequalities in the Americas: 1955-1995. J Epidemiol Community Health 2002; 56: 538541.

3- Ministério da Saúde (BR). Manual dos comitês de mortalidade materna. $3^{\mathrm{a}}$ ed. Brasília (DF); 2005.

4- Rezende CHA, Moreli D, Rezende IMAA. Mortalidade materna em cidade de médio porte, Brasil, 1997. Rev Saude Publica 2000; 34(4): 323-28.

5- Calderon IMP, Cecatti JG, Vega, CEP. Intervenções benéficas no prénatal para prevenção da Mortalidade Materna. RBGO: rev bras ginecol obstet 2006; 28(5): 310-15.

6- Albuquerque, RM; Cecatti JG; Hardy E; Faúndes A. Fatores sociodemográficos e de assistência médica associados ao óbito materno. RBG0: rev bras ginecol obstet 1998; 20(4): 181-85.

7- Oliveira RB, Melo ECP, Knupp VMAO. Perfil de óbitos infantis no município do Rio de Janeiro segundo peso ao nascer no ano de 2002. Esc Anna Nery Rev Enferm 2008 mar; 12(1): 25-9.

8- Melo ECP, Carvalho MS, Travassos C. Distribuição espacial da mortalidade por infarto agudo do miocárdio no município do Rio de Janeiro, Brasil. Cad Saude Publica 2006; 22(6): 1225-236.

9-Szwarcwald CL, Andrade CLT, Bastos FI. Income inequality, residential poverty clustering and infant mortality: a study in Rio de Janeiro, Brazil. Soc Sci Med, 2002; 55: 2083-092 bebê vive podem ser extremamente úteis. Neste sentido, a incorporação do elemento geográfico na análise dos eventos ligados à saúde permite a identificação de associações entre essa diversidade geográfica, padrões de nascimento e mortalidade, acesso aos serviços de saúde, a delimitação de áreas de abrangência das unidades do Sistema Único de Saúde, identificação do perfil de uma dada população, e associar vários fatores de risco na mesma análise. A visualização dessas relações no território vem contribuir para a formulação das ações intersetoriais capazes de consolidar políticas públicas mais integradas e efetivas voltadas para o grupo de mulheres em idade fértil.

10- Santos S, Noronha CP. Padrões espaciais de mortalidade e diferenciais sócio-econômicos na Cidade do Rio de Janeiro. Cad Saude Publica 2001; 17: 1099-110.

11- Gama SGN, Szwarcwald CL, Sabroza AR, Castelo Branco V, Leal MC. Fatores associados à assistência pré-natal precária em uma amostra de puérperas adolescentes em maternidades do município do Rio de Janeiro, 1999-2000. Cad Saude Publica 2004; 20 (sup1): S101S111.

12- Godinho RA, Schelp JRB, Parada CMGL, Bertoncello NMF. Adolescentes e grávidas: onde buscam apoio? Rev Latino-am Enfermagem 2000; 8(2): 25-32.

13- Campos TP, Carvalho MS. Assistência ao parto no município do Rio de Janeiro: perfil das maternidades e o acesso da clientela. Cad Saude Publica 2000; 16(2): 411-420.

14- Macharelli CA, Oliveira LR. Perfil do risco de óbito de crianças menores de um ano residentes em localidade do Estado de São Paulo, Brasil, 1987. Rev Saude Publica, 1991 abr; 25(2): 121-128.

15- Laguardia J. 0 uso da variável "raça" na pesquisa em saúde. PHYSIS: rev saude coletiva 2004; 14(2): 197-234.

16- Menezes DCS, Leite IC, Schramm JMA, Leal MC. Avaliação da peregrinação anteparto numa amostra de puérperas no município do Rio de Janeiro, Brasil, 1999/2001. Cad Saude Publica 2006; 22(3): 55359.

17- Sousa MH, Cecatti JG, Hardy EE, Serruya SJ. Morte materna declarada e o relacionamento de sistemas de informações em saúde. Rev Saude Publica 2007; 41(2): 181-89. 